\title{
Late onset systemic lupus erythematosus in southern Chinese
}

\author{
Carmen Tze Kwan Ho, Chi Chiu Mok, Chak Sing Lau, Raymond Woon Sing Wong
}

\begin{abstract}
Objective-Systemic lupus erythematosus (SLE) is a multisystem disorder that predominately affects women of the reproductive age. Onset of the disease beyond the age of 50 years is unusual. This study was undertaken to compare retrospectively the clinical and laboratory features between early and late onset (onset of disease beyond the age of 50 years) SLE patients in a Chinese population.

Methods-Case records of all SLE patients who attended our rheumatology clinics between 1971 and 1997 were reviewed. Patients with a disease onset beyond the age of 50 years were identified. One hundred consecutive SLE patients who had their disease onset before the age of 50 were recruited as controls. The presenting clinical features, autoantibody profile, number of major organs involved, number of major relapses, and the use of cytotoxic agents in the two groups of patients were obtained and compared.
\end{abstract}

Results-25 patients with late onset SLE were identified. All the female patients in the late onset group were postmenopausal. The female to male ratio was 3.2 to 1 , compared with 13.3 to 1 in the control group ( $p<0.02)$. Both groups had a comparable duration of disease. There were no significant differences in the presenting features between the two groups except for a lower prevalence of malar rash $(24 \% v$ $86 \%, p<0.0001$ ) and a higher prevalence of rheumatoid factor $(32 \% v 1 \%, p<0.0001)$ in the late onset patients. On subsequent visits, the late onset group had a lower prevalence of lupus nephritis $(4 \% v 51 \%$, p<0.001), fewer major organs involved (mean number of major organs involved; $0.3 v 0.9, \mathrm{p}<0.02$ ), fewer major relapses (mean number of major relapses/patient; $0.08 v 0.47, \mathrm{p}<0.002$, number of major relapses/patient year; $0.009 \quad v \quad 0.12$, $\mathbf{p}<0.001$, and required fewer cytotoxic agents for disease control (percentage of patients on cytotoxic agents; $32 \% v 79 \%$, $\mathbf{p}<0.002$ ).

Conclusion-Late onset SLE in Chinese tends to run a more benign course with fewer major organ involvement and fewer major relapses. The significantly higher incidence of male sex in late onset SLE and the milder disease course in the postmenopausal female patients suggest that oestrogen status may influence disease activity.

(Ann Rheum Dis 1998;57:437-440)
Systemic lupus erythematosus (SLE) predominantly affects women of reproductive age. It is characterised by a broad range of clinical and laboratory manifestations and is notable for a variable course, prognosis, and response to treatment. Because of such heterogeneity, attempts have been made to identify subsets of patients that would permit more accurate prediction of the course of illness in affected people. Onset of SLE beyond the age of 50 years is unusual. This group of patients has been reported to have a clinical presentation different from that seen in younger patients. This study was undertaken to compare retrospectively the clinical, laboratory, and immunological features between early and late onset SLE patients in a Chinese population.

\section{Methods}

PATIENTS

All case records of an inception cohort of SLE patients who attended our rheumatology clinics between 1971-1997 were reviewed. All patients were ethnic Chinese and fulfilled at least four of the American Rheumatology Association criteria for the classification of SLE. ${ }^{1}$ Patients with disease onset beyond the age of 50 years were identified - the late onset SLE group. One hundred consecutive case records of patients whose first disease manifestations occurred before the age of 50 years were selected as controls. All patients were followed up at least three monthly, or more frequently if necessary, for example, during a disease flare. The presenting clinical features, autoantibody profile, number of major organs involved, number of major relapses, and the use of immunosuppressive agents in both groups of patients were recorded. Major organ involvement was defined as involvement of an organ/ system to the extent that high dose prednisolone $(>0.5 \mathrm{mg} / \mathrm{kg} /$ day $)$ with or without cytotoxic agents were needed for disease control. These included nephritis (WHO class III-V), severe serositis, neuropsychiatric lupus, transverse myelitis, pulmonary haemorrhage, severe thrombocytopenia $(<20$ $\times 10^{9} / 1$ ), and haemolytic anaemia. Joint and skin diseases were not included. A major relapse was defined as a severe disease flare that was accompanied by the need to start or augment the dose of prednisolone to more than $0.5 \mathrm{mg} / \mathrm{kg} /$ day with or without the subsequent use of cytotoxic agents, based on clinical grounds. 
Table 1 Frequency of clinical manifestations and laboratory findings at presentation and during follow up in late onset $(n=25)$ and control $(n=100)$ lupus patients

\begin{tabular}{|c|c|c|c|c|c|c|}
\hline \multirow[b]{2}{*}{ Clinical features } & \multicolumn{3}{|c|}{ Initial presentation } & \multicolumn{3}{|c|}{ During follow up } \\
\hline & $\begin{array}{l}\text { late onset } \\
n(\%)\end{array}$ & $\begin{array}{l}\text { control } \\
n(\%)\end{array}$ & $p$ value & $\begin{array}{l}\text { late onset } \\
n(\%)\end{array}$ & $\begin{array}{l}\text { control } \\
n(\%)\end{array}$ & $p$ value \\
\hline Malar rash & $6(24)$ & $86(86)$ & $<0.0001$ & $7(28)$ & $86(86)$ & $<0.0001$ \\
\hline Discoid rash & $0(0)$ & $8(8)$ & 0.1 & $0(0)$ & $8(8)$ & 0.31 \\
\hline Photosensitivity & $2(8)$ & $26(26)$ & 0.10 & $2(8)$ & $27(27)$ & 0.08 \\
\hline Serositis & $2(8)$ & $8(8)$ & 0.68 & $3(12)$ & $8(8)$ & 0.81 \\
\hline Oral ulcer & $5(20)$ & $8(8)$ & 0.16 & $5(20)$ & $9(9)$ & 0.23 \\
\hline Arthritis & $19(76)$ & $86(86)$ & 0.36 & $21(84)$ & $86(86)$ & 0.95 \\
\hline Alopecia & $4(16)$ & $26(26)$ & 0.43 & $4(16)$ & $26(26)$ & 0.43 \\
\hline Keratoconjunctivitis sicca & $2(8)$ & $1(1)$ & 0.19 & $3(12)$ & $1(1)$ & 0.03 \\
\hline Raynaud's phenomenon & $1(4)$ & $8(8)$ & 0.80 & $2(8)$ & $8(8)$ & 0.68 \\
\hline Fever & $10(40)$ & $18(18)$ & 0.02 & $10(40)$ & $18(18)$ & 0.02 \\
\hline Myalgia & $0(0)$ & $7(7)$ & 0.38 & $1(4)$ & $7(7)$ & 0.93 \\
\hline CNS involvement* & $0(0)$ & $4(4)$ & 0.70 & $0(0)$ & $4(4)$ & 0.70 \\
\hline Nephritis† & $0(0)$ & $9(9)$ & 0.26 & $1(4)$ & $51(51)$ & $<0.001$ \\
\hline Pancytopenia $\ddagger$ & $1(4)$ & $5(5)$ & 0.75 & $1(4)$ & $5(5)$ & 0.75 \\
\hline Lymphopenia $\ddagger$ & $9(36)$ & $38(38)$ & 0.85 & $11(44)$ & $38(38)$ & 0.58 \\
\hline Haemolytic anaemia & $3(12)$ & $20(20)$ & 0.53 & $5(20)$ & $20(20)$ & 0.78 \\
\hline Thrombocytopenia $\ddagger$ & $4(16)$ & $25(25)$ & 0.49 & $5(20)$ & $25(25)$ & 0.79 \\
\hline Anti-nuclear antibody & $25(100)$ & 99 (99) & 0.45 & $25(100)$ & $99(99)$ & 0.45 \\
\hline Anti-dsDNA antibody & $18(72)$ & $79(79)$ & 0.45 & $21(84)$ & $79(79)$ & 0.78 \\
\hline Anti-Ro antibody & $15(60)$ & $51(51)$ & 0.42 & $15(60)$ & $53(53)$ & 0.53 \\
\hline Anti-La antibody & $2(8)$ & $8(8)$ & 0.68 & $2(8)$ & $9(9)$ & 0.81 \\
\hline Anti-Sm antibody & $5(20)$ & $9(9)$ & 0.23 & $6(24)$ & $9(9)$ & 0.09 \\
\hline Anti-RNP antibody & $5(20)$ & $17(17)$ & 0.95 & $5(20)$ & $19(19)$ & 0.86 \\
\hline Rheumatoid factor & $8(32)$ & $1(1)$ & $<0.001$ & $8(32)$ & $1(1)$ & $<0.0001$ \\
\hline
\end{tabular}

${ }^{\star} \mathrm{CNS}=$ central nervous system, referring to neuropsychiatric involvement based on the $1982 \mathrm{ACR}$ revised criteria of $\mathrm{SLE}^{1}$ (that is, psychosis and seizure). + Nephritis $=$ biopsy confirmed lupus nephritis. $\neq$ Pancytopenia $=$ total leucocyte count $<3.5 \times 10^{9} / 1$ plus haemoglobin $<11 \mathrm{~g} / \mathrm{dl}$ plus platelet count $<130 \times 10^{9} / 1$ on at least two occasions. Lymphopenia $=$ peripheral lymphocyte count $<1.5 \times 10^{9} / 1$ on at least two occasions. Thrombocytopenia $=$ platelet count $<130 \times 10^{9} / 1$ on at least two occasions. Anti-nuclear antibodies were detected by indirect immunofluorescence. Anti-dsDNA antibody was detected by the Crithidia assay before 1992, and ELISA since then. Anti-ENA antibodies were detected using the counter-current immunoelectrophoresis. Rheumatoid factor was assayed by latex agglutination before 1991, and ELISA since then.

STATISTICAL ANALYSIS

Comparison of non-parametric data between the two groups of patients was made by using the $\chi^{2}$ test. Fisher's exact test was used whenever the number was small. For comparison of parametric data, the Student $t$ test was adopted. Because of multiple comparisons, only differences at $\mathrm{p}<0.01$ were considered statistically significant.

\section{Results}

Three hundred case records were reviewed. Ten were excluded because of missing or incomplete case notes. From the remaining pool of patients, 100 consecutive SLE patients who had a disease onset before the age of 50 were selected as controls for comparison. The mean (SD) age of onset of SLE for the control and late onset groups were 28.3 (8.5) years (range: 13-47) and 63.3 (10.6) years (range: $51-86)$, respectively $(\mathrm{p}<0.0001)$. The female/ male ratio of the control patients was 13.3:1 (93 females and 7 males) and that of the late onset group was 3.2:1 (19 females and 6 males, $\mathrm{p}<0.02)$. All our female late onset SLE patients were postmenopausal and none of them were receiving hormonal replacement therapy. The mean time interval between the onset of symptoms to the diagnosis of SLE was shorter in the controls (1.7 (3.2) months) than the late onset patients (5 (14.2) months) but the difference did not reach statistical significance. The duration of disease was comparable in the two groups of patients (5.6 (4.4) years for the controls and 5.7 (5.5) years for the late onset SLE patients, $\mathrm{p}=1.00$ ).

Table 1 lists the frequency of SLE clinical manifestations and laboratory findings at presentation and during subsequent follow up for the two groups of patients. There were no significant differences in clinical features at presentation between the two groups except that control patients had a significantly higher incidence of malar rash $(86 \% \quad v 24 \%$, $\mathrm{p}<0.0001)$ and lower incidence of rheumatoid factor $(1 \% v 32 \%, \mathrm{p}<0.0001)$. During follow up, late onset SLE patients had a significantly lower incidence of biopsy confirmed lupus nephritis $(4 \%$ v $51 \%, \mathrm{p}<0.001)$.

Late onset lupus patients had significantly less major organ involvement when compared with controls (mean number of major organs involved/patient in the late onset group and controls were 0.3 and 0.9 , respectively; $\mathrm{p}<0.02)$. The mean number of major relapses per patient $(0.08$ in late onset group $v 0.47$ in controls; $\mathrm{p}<0.002$ ) and number of major relapses per patient year of follow up $(0.009 \mathrm{v}$ $0.12 ; \mathrm{p}<0.001)$ were also significantly lower in this group. Use of cytotoxic agents for disease control was less likely in the late onset than the control group (32\% v 79\%, p<0.002). Seven $(28 \%)$ patients in the late onset group and 61 (61\%) patients in the control group were given azathioprine, and one $(4 \%)$ patient in the late onset group and $18(18 \%)$ patients in the control group were given cyclophosphamide for the treatment of class IV nephritis.

Two late onset SLE patients died during the study period. One died at the age of 69 of subarachnoid haemorrhage, which was unrelated to her lupus disease. The other, aged 76, died of septicaemia complicating a perforated bowel. Whether the second death was related to the lupus process was not clear because the patient's relatives declined a postmortem examination. None died in the control group during the study period. 


\section{Discussion}

Many previous studies ${ }^{2-9}$ have suggested that late onset SLE patients differ from those with early onset in clinical presentation, pattern of organ involvement, severity of disease, and prognosis. Additionally, there may be inter-ethnic differences in some of these clinical features. SLE is relatively common among Chinese in Hong Kong. ${ }^{10}$ However, there has so far been only one publication ${ }^{11}$ on late onset lupus in Chinese patients. Our main objective was therefore to determine whether the above concept holds for our Chinese population.

Our study is in accordance with most previously reported series of a less pronounced female predominance in SLE patients whose disease first presented later in life..$^{2-911} 12$ This leads to the postulation that female sex hormones may play a part in determining the expression of the disease. Lupus may appear, or the disease may exacerbate, when serum concentrations of reproductive hormones change rapidly. In the pre-corticosteroid era, improvement was noted after the patient had reached menopause or had surgical removal of the uterus and ovaries. ${ }^{13}$ This suggests that oestrogen status may be important in determining disease activity in SLE.

In our study, the interval between symptom onset and the diagnosis of SLE was found to be longer in the late onset group than the controls, although the difference did not reach statistical significance. Similar results have been suggested previously. ${ }^{2459}$ This illustrates that late onset SLE patients may present atypically at disease onset, leading to a delay in diagnosis. In fact, six of our patients fulfilled only three ARA criteria for SLE at presentation, when the diagnosis was uncertain. Four of them developed more features of SLE on follow up while two remained labelled as having lupus-like disease because no new features evolved. These two patients had positive ANA, positive anti-dsDNA, anti-Ro and thrombocytopenia and had been excluded from our analysis.

The clinical presentation of late onset SLE patients varies in different series. Commonly reported clinical features include fever, weight loss, musculoskeletal complaints, arthritis, oral ulcers, and pleuropericarditis. Wilson et $a l^{\beta}$ and Maddison ${ }^{5}$ also described an increased prevalence of Raynaud's phenomenon, interstitial lung disease, and cutaneous and neuropsychiatric involvement in elderly lupus patients. Late onset lupus patients in the present study had a significantly lower incidence of malar rash and lupus nephritis when compared with younger lupus patients. Raynaud's phenomenon was less common although this might be related to the warmer climate in Hong Kong. In late onset lupus patients, there appeared to be a higher prevalence of sicca complex $(12 \% v 1 \%)$ though the difference was not statistically significant. Only Maddison ${ }^{5}$ and Catoggio et $a l^{7}$ had reported the frequency of sicca complex (42\% and $38 \%$, respectively) in their studies. As our study was conducted retrospectively, underes-
Table 2 Percentage frequency of auto-antibody profile in different series of late onset lupus patients

\begin{tabular}{|c|c|c|c|c|c|c|c|}
\hline & $A N A$ & $D N A$ & $R F$ & $R o$ & $L a$ & $R N P$ & $S m$ \\
\hline $\begin{array}{c}\text { Gossat } e t a l^{4} \\
(\mathrm{n}=14)\end{array}$ & 93 & 93 & NA & NA & NA & NA & NA \\
\hline $\begin{array}{l}\text { Maddison } \\
\quad(n=19)\end{array}$ & 95 & 53 & 63 & 84 & 63 & 0 & 0 \\
\hline $\begin{array}{l}\text { Catoggio et al } \\
\quad(\mathrm{n}=13)\end{array}$ & 85 & 31 & 85 & 92 & 61 & 0 & 0 \\
\hline $\begin{array}{l}\text { Koh et al } \\
\quad(\mathrm{n}=26)\end{array}$ & 96.2 & 84.6 & 23.5 & 34.8 & 7.7 & $\begin{array}{ll}7 & 17.4\end{array}$ & 21.7 \\
\hline $\begin{array}{l}\text { Present study } \\
\quad(n=25)\end{array}$ & 100 & 86 & 32 & 60 & 8 & 20 & 24 \\
\hline
\end{tabular}

timation could not be avoided and confirmatory tests (for example, Schirmer's test and salivary gland biopsy) were not performed in some of the patients. Therefore, the true prevalence of Sjogren's syndrome could have been higher.

Table 2 shows a comparison of the autoantibody profile of late onset lupus patients from five different studies including this one. As can be noted, anti-Ro and anti-La antibodies were more commonly found in white patients ${ }^{5-7}$ while Chinese patients showed a higher frequency for anti-Sm and anti-nRNP antibodies. It should, however, be noted that some of these differences may have partially been related to different procedures used to assay the various antibodies. Among our patients, the autoantibody profile of those with late disease onset did not differ significantly from that of control patients. Only RF was more commonly found in the late onset group. This could be explained by the fact that RF is more commonly found in the older population. Despite the high prevalence of anti-dsDNA (which was comparable to the control group) in our late onset SLE patients, they had fewer major organs involved when compared with the younger lupus patients. In addition, late onset SLE patients had a milder disease course, which was reflected by the significantly lower mean number of major relapses per patient, number of major relapses per patient year, and that fewer patients required the use of cytotoxic agents for disease control during the course of their disease.

In conclusion, our retrospective study of Chinese SLE patients showed differences in disease onset, pattern of organ involvement, and serology between the late and early onset groups. Late onset SLE patients have a milder disease course with fewer major organs involved, fewer major relapses, and less aggressive immunosuppression required for disease control. Prospective studies involving a larger number of late onset SLE patients are needed to confirm whether they constitute a distinct subset of SLE regarding clinical presentation and prognosis.

1 Tan EM, Cohen AS, Fries JF, Masi AT, McShane DJ, Rothfield NF, et al. The 1982 revised criteria for the classification of systemic lupus erythematosus. Arthritis Rheum 1982;25:1271-7.

2 Foad BSI, Sheon RP, Kirsner AB. Systemic lupus erythematosus in the elderly. Arch Intern Med 1972:130: 743-6.

3 Wilson HA, Hamilton ME, Spyker DA, Brunner CM, O'Brien WM, Davis JS, et al. Age influences the clinical and serologic expression of systemic lupus erythematosus. Arthritis Rheum 1981:24:1230-5. 
4 Gossat DM, Walls RS. Systemic lupus erythematosus in later life. Med J Aust 1982:1:297-9.

5 Maddison PJ. Systemic lupus erythematosus in the elderly. J Rheumatol 1987:14:182-7.

6 Jonsson H, Nived O, Sturfelt G. The effect of age on clinical and serological manifestations in unselected patients with systemic lupus erythematosus. J Rheumatol 1988:15: 505-9.

7 Catoggio LJ, Skinner RP, Smith G, Maddison PJ. Systemic lupus erythematosus in the elderly: Clinical and serological characteristics. J Rheumatol 1984:11:175-81.

8 Ward MM, Polisson RP. A meta-analysis of the clinical manifestations of older onset systemic lupus erythematosus. Arthritis Rheum 1989:32:1226-32.
9 Font J, Pallares L, Cervera R, Lopez-Soto A, Navarro M, Bosch X, et al. Systemic lupus erythematosus in the elderly: clinical and immunological characteristics. Ann Rheum $1.50: 702-5$

10 Lee SS, Li CS, Li PC. Clinical profile of Chinese patients with systemic lupus erythematosus. Lupus 1993;2:105-9. 11 Koh ET, Boey ML. Late onset lupus: A clinical and immunological study in a predominantly Chinese population. J Rheumatol 1994:21:1463-7.

12 Joseph RR, Zarafonetis CJD. Clinical onset of lupus erythematosus in the older age group. J Am Geriatr Soc 1964:12:787-99.

13 Rose E, Pillsbury DM. Lupus erythematosus and ovarian function: observation on a possible relationship, with report of six cases. Ann Intern Med 1944;21:1022-34.

\section{Annals of the Rheumatic Diseases - http://www.annrheumdis.com}

Visitors to the world wide web can now access Annals of the Rheumatic Diseases either through the BMJ Publishing Group's home page (http://www.bmjpg.com) or directly by using its individual URL (http:// www.annrheumdis.com). There they will find the following:

- Current contents list for the journal

- Contents lists of previous issues

- Members of the editorial board

- Subscribers' information

- Instructions for authors

- Details of reprint services

A hotlink gives access to:

- BMJ Publishing Group home page

- British Medical Association web site

- Online books catalogue

- BMJ Publishing Group books

The web site is at a preliminary stage and there are plans to develop it into a more sophisticated site. Suggestions from visitors about features they would like to see are welcomed. They can be left via the opening page of the BMJ Publishing Group site or, alternatively, via the journal page, through "about this site". 